\section{Response to Letter To THE Editor}

\section{RE: Intracranial Pressure Monitors in Traumatic Brain Injury: A Systematic Review. Can J Neurol Sci. 2013;40: 433-434.}

We thank Dr. Figaji for his thoughtful commentary ${ }^{1}$ on our recent systematic review addressing intracranial pressure monitors in traumatic brain injury (TBI $)^{2}$. We completely concur with his eloquent summary of the limitations of the literature. Although Dr. Figaji takes slight issue with our contention that in order to improve outcome, intracranial pressure (ICP) monitoring "... must be acted upon in a standardized and reproducible manner", we feel our statement is in fact congruous with his central thesis. This is illustrated nicely in his discussion: elevated ICP is simply a number which may represent various pathophysiologic mechanisms which themselves may have very different treatment approaches.

Intracranial pressure monitoring may be considered analogous to the pulmonary artery catheter (PAC) which generates objective physiologic information which the clinician must then interpret to both diagnose the problem and prescribe a remedy. However, the "right" response is predicated on correct interpretation by clinicians, who perform poorly in this regard ${ }^{3}$. Previous trials on PAC use have been criticized for both lack of training of study personnel on variable interpretation and lack of treatment algorithms in response to PAC data ${ }^{4}$. Because interventions (in this case ICP monitors) generally have small treatment effects, standardization of clinical decisions is requisite for rigorous scientific evaluation ${ }^{5}$. Dr. Figaji illustrates the challenges with developing a standardized algorithm to approach ICP monitoring. However, standardization of care has resulted in improved outcomes in other complex systems, including patients with $\mathrm{TBI}^{6}$. Hopefully neuromonitoring will lead to an improved understanding of the pathophysiologic mechanisms underlying our this complex disease process. As always, the goal is to improve care for our patients, something we can all agree on.

Donald E. G. Griesdale ${ }^{1,3-5}$, Asher A. Mendelson ${ }^{1}$, Chris Gillis ${ }^{2}$, William R. Henderson ${ }^{1,3}$, Juan J. Ronco ${ }^{1,3}$, Vinay Dhingra ${ }^{1,3}$

University of British Columbia ${ }^{1,2}$; Vancouver General Hospital ${ }^{3,4}$; Vancouver Coastal Health Research Institute ${ }^{5}$ Vancouver, British Columbia, Canada Email:donald.griesdale@vch.ca

\section{REFERENCES}

1. Figaji A. ICP monitoring - interpreting the literature and evaluating practice. Can J Neurol Sci. 2013;40(3):433-4.

2. Mendelson A, Gillis C, Henderson WR, Ronco JJ, Dhingra V, Griesdale DEG. Intracranial pressure monitors in traumatic brain injury: a systematic review. Can J Neurol Sci. 2012 Sep;39(5): 571-6.

3. Squara P, Bennett D, Perret C. Pulmonary artery catheter: does the problem lie in the users? Chest. 2002 Jun;121(6):2009-15.

4. Shure D. Pulmonary-artery catheters-peace at last? NEJM. 2006; 354(21):2273-4

5. Morris AH. Developing and implementing computerized protocols for standardization of clinical decisions. Ann Intern Med. 2000 Mar 7;132(5):373-83

6. Patel HC, Menon DK, Tebbs S, Hawker R, Hutchinson PJ, Kirkpatrick PJ. Specialist neurocritical care and outcome from head injury. Intensive Care Med. 2002 May;28(5):547-53.

\section{To THE EDITOR}

\section{Bacillus Thuringiensis Poisoning Related Acute Transverse Myelitis}

A 25-year-old Asian woman with acute spinal shock was referred to our institution for therapy. She attempted suicide by drinking $250 \mathrm{mg}$ of insecticide containing Bacillus thuringiensis (8000IU/mg) seven days prior to admission. She felt nausea soon after drinking the insecticide, but did not vomit nor did she go to see the doctor until five days later when she felt headache, abdominal pain, sudden weakness on her both legs, and was unable to urinate.

On clinical examination, we found the patient to have normal temperature, pulse, blood pressure and respiration, without mental disorders. The patient experienced a stiff neck. Neurologic evaluation revealed paresis of both lower limbs of grade $0 / 5$ and hypotonia, without Babinskii's sign, knee and ankle deep tendon reflexes were elicited, T10-level sensory deficit, impaired joint position sensation of the lower limbs, and absence of anal sphincter tone. Her cranial nerve functions were normal.
Cerebrospinal fluid (CSF) analysis showed translucent fluid with an opening pressure of $300 \mathrm{cmH}_{2} \mathrm{O}$. The CSF glucose, chloride and adenylate deaminase contents were normal and CSF protein was as high at $98 \mathrm{mg} / \mathrm{dL}$. The CSF leukocyte count was high $\left(220 \times 10^{6} / \mathrm{L}\right.$, of which 97 percent was neutrophils and 3 percent was lymphocytes). The CSF erythrocyte count was also high $\left(440 \times 10^{6} / \mathrm{L}\right)$, however, traumatic puncture was denied and CSF culture for bacteria and fungus showed negative results.

Her serum immunological examination and peripheral blood tests were normal. Tests for antibodies of Coxackie virus, CMV, EBV, HBV, HCV, HIV, HSV1, HSV2, VZV, bacillus tuberculosis, syphilis in serum and CSF, as well as tumor markers in serum revealed no etiological values. Cultures of her urine, stool, and blood were all negative.

Her first spinal magnetic resonance imaging (MRI), taken on the onset day of paralysis, which was five days after her suicide attempt, was normal (Figure 1). Her second spinal MRI, which was taken eight days after her suicide attempt, indicated diffused abnormities in whole spinal cord, along with severe swelling of spinal cord (Figure 2). Cerebral MRI was normal.

Electroencephalogram and visual evoked potentials were normal. Electromyography and peripheral nerve conduction velocity both indicated upper-neuron damage of all four limbs. 


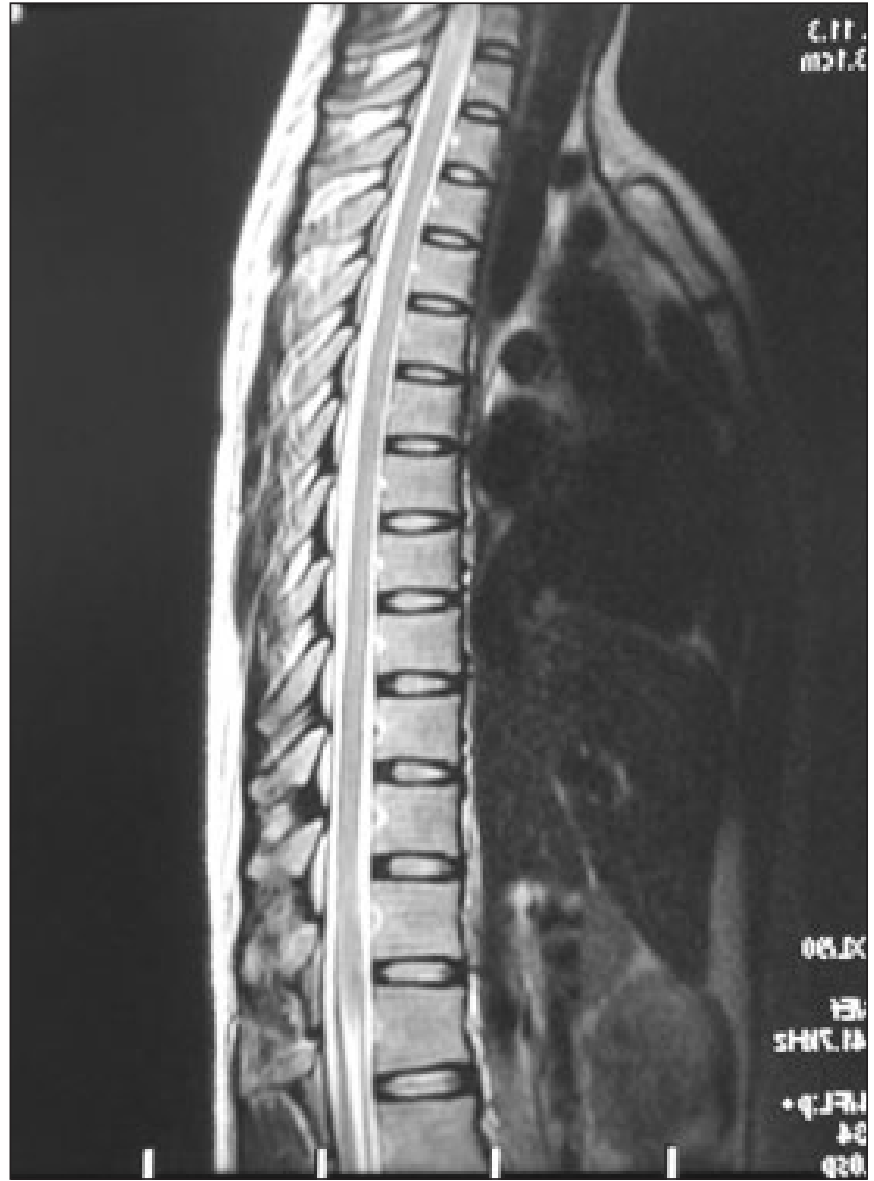

Figure 1: A T2-weighted sagittal view of thoracic and lumbar spine MRI of the patient, taken on the day of onset, (five days post suicide attempt), was normal.

After admission, the patient kept suffering from severe abdominal pain, accompanied by poor appetite, and her feces test indicated continuous bloody stools. Her abnormal sensory level kept ascending, and weakness gradually spread to her upper limbs.

Three weeks of treatment with intravenous antibiotics, cortisone, mannitol and vitamin B had no effects.

The levels of anesthesia and paralysis kept ascending. Four weeks after onset, her levels of anesthesia stayed at T4, and both upper limbs were force less of grade $2 / 5$ and hypotonia, without respiratory muscle injuries. She was transferred for rehabilitation exercise. One year of follow-up revealed no improvement of her disabilities.

\section{Discussion}

As a final diagnosis, we considered the most likely one to be acute transverse myelitis resulting from Bacillus thuringiensis poisoning. The clinical diagnosis was based on the Transverse Myelitis Consortium Working Group Criteria for Idiopathic Acute Transverse Myelitis. The etiological diagnosis was based on the history of intaking high doses of Bacillus thuringiensis. Other common etiologies, such as viral infection, syphilis, tuberculosis, multiple sclerosis, vertebral dislocation, spinal cord hemorrhage, tumor, spinal-contusion, spinal-vascular malformation, serologic or clinical evidence of connective tissue diseases were all excluded. Although we did not finish the specific tests of Bacillus thuringiensis or Cry toxins in blood or CSF, the accordance of onset time with swallowing pesticides highly indicated that poisoning was the etiology.

After consulting the literature, we think this case of acute transverse myelitis based on Bacillus thuringiensis poisoning is the first ever described in humans.

Bacillus thuringiensis is an aerobic, gram-positive, sporeforming bacterium from the Bacillus cereus group. They can

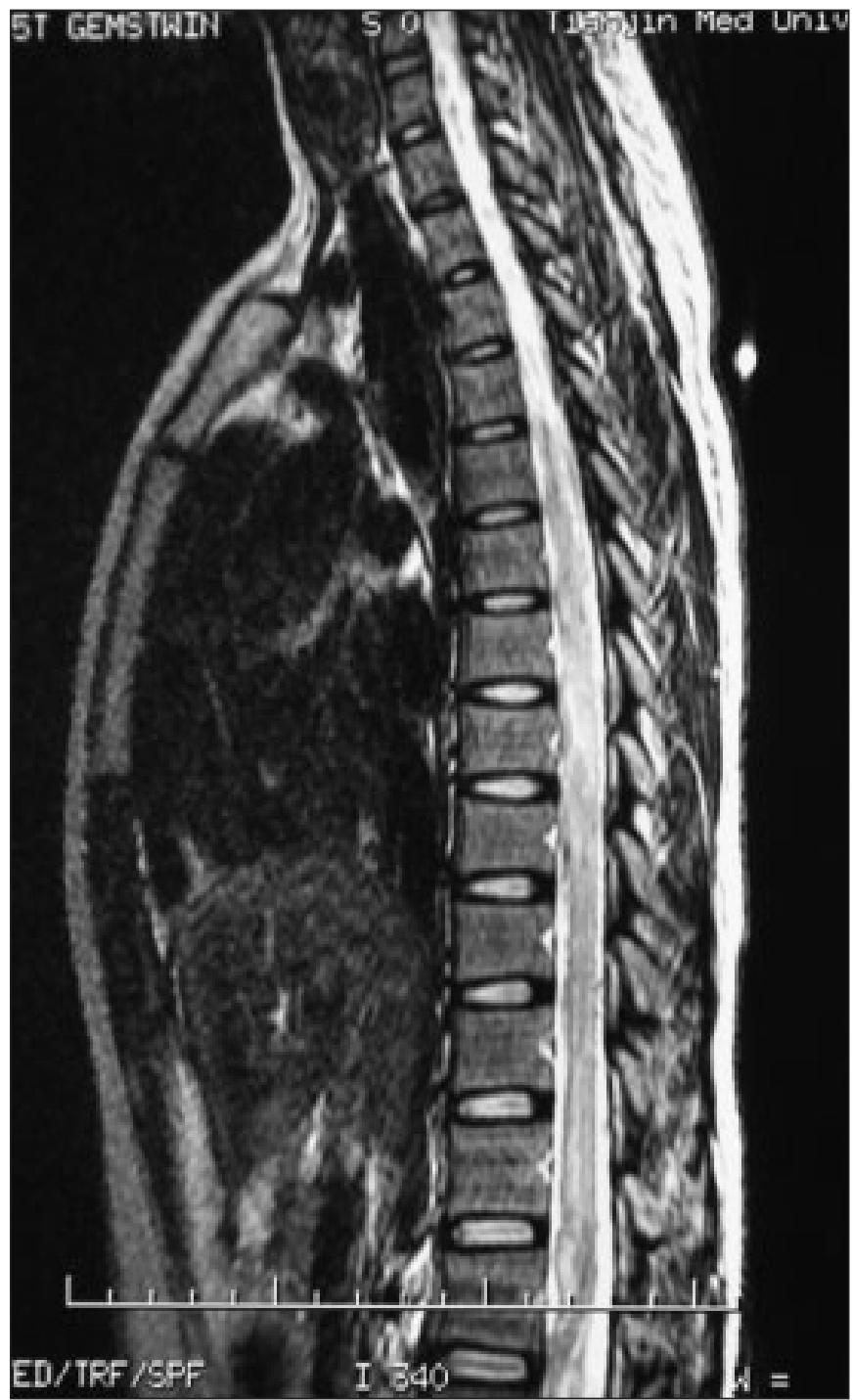

Figure 1: A T2-weighted sagittal view of thoracic and lumbar spine MRI of the patient, taken 3 days after the Figure 1, ( 8 days post her "attempted" suicide), which showed diffused hyper-signal intensity from upper cervical to lower lumbar spinal cord, along with severe swelling of whole spinal cord. 
produce Cry toxins (delta-endotoxins) during the sporulation phase, which are pore-forming toxins that have insecticide activity. One feature that distinguishes Cry toxins is their remarkable specificity, and they are therefore harmless to nontarget insects and animals ${ }^{1}$. Nowadays, they are widely used in insect control in agriculture and forestry, in the control of mosquito-borne human diseases, and in the development of transgenic insect resistant plants. Based on former laboratory studies, field experience and epidemiology studies, it has been widely accepted that products of Bacillus thuringiensis are safe to humans, animals, and the environment.

However, some recent laboratory studies put the safety of the pesticides in question. According to Cerstiaens's study, one of the Cry toxins, Cry1C, has been proved to be toxic to primary cultured neurons of Lymantria, in a concentration of $20 \mathrm{ug} / \mathrm{ml}$ in vitro, ${ }^{2}$. Likewise, it is possible that the insecticides also possess neurotoxicity to humans. On the other hand, a recent study addressed the effect of sub-lethal doses of biological and synthetic pesticides of Bacillus thuringiensis on female rats' reproduction apparatus during pregnancy; no miscarriages or malformations of the neonates occurred. But the pups, who received more or less of the insecticide, produced similar lesions in the kidneys, livers and lungs and had reduced fertility. This study suggested that sublethal doses of insecticides possibly provide chronic toxicity to humans ${ }^{3}$.

There are only two literature reports of Bacillus thuringiensis infection in man between the year 1997 and the present, and all infected individuals had experienced either extensive burns or a blast injury, which predisposed them to infection ${ }^{4}$. Based on the literature, the symptoms of Bacillus thuringiensis infection or poisoning included mild irritative pulmonary symptoms, theoretical risk of respiratory infection in immunocompromised individuals, single corneal ulceration, mild gastroenteritis with heavy ingestion ${ }^{5}$.

In this case, the patient had no vomiting, diarrhea or gastrolacage after swallowing the pesticides. We speculated that, high doses of insecticides were absorbed through the gastrointestinal tract, which secondarily induced diffused spinal cord injury. However, the specific mechanisms of this injury are unknown. It is possible that Cry toxins directly attacked neurons and lead eventually to cell death. Alternatively, the toxins possibly induced auto-immune responses against neurons.

We know that most poisonings from pesticides do not have a specific antidote. Therefore, decontamination is the most effective intervention. Unfortunately, the patient missed the best chance for treatment, which lead to her bad prognosis.

In conclusion, few human tests with high dose of Bacillus thuringiensis have been performed. In line with the increased spread of the use of such pesticides in agriculture and transgenic plants, the side effects of their long term applications deserve more consideration.

Jing Han, Yan Cheng, Mei-sheng Wang Department of Neurology, Tianjin Neurology Institute, Tianjin Medical University General Hospital, 154 Anshan Road Heping District, Tianjin 300052, China Email:quartz945@163.com

\section{REFERENCES}

1. Bravo A, Gill SS, Soberon M. Bacillus thuringiensis mechanism and use. In: Gilbert LI, Iatrou K, Gill SS, editors. Comprehensive molecular insect science. Elservier B.V.; 2005. p. 175-205.

2. Cerstiaens A, Verleyen P, Van Rie J, et al. Effect of Bacillus thuringiensis Cry1 toxins in insect Hemolymph and their neurotoxicity in brain cells of Lymantria dispar. Appl Environ Microbial. 2001;67(9):3923-7.

3. Lemos AJ, Siqueira HA, Wanderley-Teixeira V, et al. Effect of sublethal doses of Bacillus thuringiensis subsp. Aizawai and deltamethrin with regard to fertility and organ toxicity in pregnant albino rats. Exp Toxicol Pathol. In Press. 2012.

4. Siegel JP. The mammalian safety of Bacillus thuringiensis-based insecticides. J Invertebr Pathol. 2001;77(1):13-21.

5. Simpson WM Jr, Schuman SH. Recognition and management of acute pesticide poisoning. Am Fam Physician. 2002;65(8): 1599-604.

\section{TO THE EDITOR}

\section{Cerebral Amyloid Angiopathy Presenting with TIA-like Episodes}

Cerebral amyloid angiopathy (CAA) is a common age-related cerebral small vessel disease characterized by a progressive deposition of amyloid- $\beta$ in the wall of cortical and leptomeningeal small arteries, with or without capillary involvement. It is a frequent cause of spontaneous intracerebral hemorrhage and cognitive impairment in the elderly ${ }^{1}$. Another characteristic clinical presentation associated with CAA is transient focal neurological episode (TFNE). As recently reported, TFNE could be divided into a positive-symptoms form (aura-like) and a negative-symptoms form (transient ischemic attack-like $)^{2}$.

We present a case of probable cerebral amyloid angiopathy presenting with transient ischemic attack-like episodes.

\section{Case presentation}

A 69-year-old Caucasian man was admitted to the emergency department because of acute onset right arm weakness and speech impairment, lasting 30 minutes. A computed tomogram (CT) brain scan showed leukoaraiosis and mild cortical atrophy. The patient complained of a similar episode one day before. He was a smoker and moderate alcohol drinker; he had referred with higher blood pressure and mild carotid atherosclerosis for two years. He was taking ramipril, aspirin $100 \mathrm{mg}$ and low-dose statin. ABCD2 score was moderate. At the admission to the Stroke Unit his blood pressure was $130 / 80 \mathrm{mmHg}$, heart rate was sixty rhythmic, and the neurological examination was normal. Epiaortic ultrasound study showed mild atherosclerosis; Transcranial doppler (TCD) ultrasound was normal as well as CT cerebral angiography. We thought transient ischemic attacks (TIA) episodes and started higher dose antiaggregation with aspirin $300 \mathrm{mg}$ and atorvastatin $80 \mathrm{mg}$. The day after, the patient 